Revue d'histoire de l'Amérique française

ALE REVUE D.HISTOIRE DE L'AMÉRIQUE FRANÇAISE

\title{
Les structures de l'Église et la conjoncture coloniale en Nouvelle-France, 1632-1674
}

\section{Jean Blain}

Volume 21, numéro 4, mars 1968

URI : https://id.erudit.org/iderudit/302722ar

DOI : https://doi.org/10.7202/302722ar

Aller au sommaire du numéro

Éditeur(s)

Institut d'histoire de l'Amérique française

ISSN

0035-2357 (imprimé)

1492-1383 (numérique)

Découvrir la revue

Citer cet article

Blain, J. (1968). Les structures de l'Église et la conjoncture coloniale en Nouvelle-France, 1632-1674. Revue d'histoire de l'Amérique française, 21(4), 749-756. https://doi.org/10.7202/302722ar d'utilisation que vous pouvez consulter en ligne.

https://apropos.erudit.org/fr/usagers/politique-dutilisation/ 


\section{LES STRUCTURES DE L'ÉGLISE ET LA CONJONCTURE COLONIALE EN NOUVELLE-FRANCE, 1632-1674*}

L'Église de la Nouvelle-France, son orientation, ses structures ont été visiblement marquées par les transformations qu'ont connues la politique et la réalité coloniales de 1632 à 1675 . En ce sens, elle s'imbrique assez bien comme institution dans la conjoncture socio-politique qui, à chaque tournant, lui dessine la voie qu'elle doit suivre.

Quand les Français reviennent à Québec en 1632, c'est une colonie de peuplement qu'ils entendent mettre sur pied à la suite des vœux émis et réitérés par Champlain et les missionnaires depuis les années 1615. Créée dans cette optique, la Compagnie de la Nouvelle-France est un instrument grâce auquel on espère intégrer la vieille colonie d'exploitation qui existe depuis la dernière moitié du $\mathrm{XVI}^{\mathrm{e}}$ siècle dans un ensemble d'activités dont le moteur serait un noyau de société prenant racine dans la vallée du Saint-Laurent.

Or il se trouve que cette société embryonnaire, numériquement bien moins importante qu'on l'avait souhaité, présente comme caractéristique, dès le début, ce que l'on pourrait appeler l'hypertrophie $d u$ sens et de la fonction missionnaires. La proportion extrêmement élevée par rapport à l'ensemble de la population du nombre des religieux et des religieuses venus au Canada dans le but avoué de s'occuper de la conversion des Indiens suffit à l'établir.

Mais il y a plus, les immigrants qu'on évalue au nombre d'environ un millier de 1632 à 1663, sont eux-mêmes liés directement ou indirectement, pour une portion peut-être majoritaire

* Conclusion d'une thèse de doctorat intitulée L'Eglise de la NouvelleFrance, 1632-1675. La mise en place des structures, présentée avec succès à l'Université d'Ottawa, le 5 octobre 1967. 
d'entre eux, à la mystique missionnaire. De ce nombre, il faut compter les ouvriers, les serviteurs, les "donnés" à l'emploi des communautés religieuses, les censitaires (qu'on peut croire triés sur le volet) établis dans leurs seigneuries et, surtout, le contingent appréciable des Montréalistes.

Cette petite société correspond assez bien à la politique et à la propagande explicitées par les Relations des Jésuites à partir de 1632. Pour les missionnaires, en effet, la colonisation de la Nouvelle-France est une entreprise d'apostolat et de salut personnel. Elle doit, par l'exemple des colons rivés à la terre, contribuer à l'enracinement et à l'occidentalisation - bien dosée - des populations indigènes qui seront par là-même mieux orientées sur la voie de la vérité évangélique. Mais elle doit aussi fournir aux immigrants l'occasion de préparer un salut éternel qui sera d'autant mieux assuré que le cheminement en aura été pénible dans ce monde primitif à bâtir, loin des douceurs, des raffinements et des excès du siècle. A cet égard, l'attaque incessante et cruelle de l'Iroquois n'est pas loin d'être considérée comme un bienfait du Ciel qui permet à quelques privilégiés d'acquérir la palme convoitée du martyre.

La mystique n'est pas que canadienne. Elle est soutenue et alimentée en France par une génération d'hommes de bien et de dévots qui a redécouvert l'idéal de la propagation de la foi et pour qui le Canada est une terre d'expérimentation rêvée. Ce sont ces esprits fervents qui, par-delà les rouages administratifs officiels, ont contribué le plus, entre 1632 et 1663, à maintenir en place dans la région Montréal-Québec les bases fragiles d'une colonie de peuplement que la conjoncture économique ne pouvait justifier.

Bien sûr, l'apostolat chez les infidèles, le renoncement à soi, le rêve d'une cité missionnaire, c'est dans les écrits des Jésuites, des Ursulines, des fondateurs de Montréal qu'on les retrouve et pas ailleurs. Mais se peut-il que le peuple qui vivait sous leur tutelle non seulement religieuse, mais encore intellectuelle, sociale et même politique, qui, pour une bonne part, avait immigré au Canada pour répondre à leurs appels, qui ne trouvait 
sur place aucune incitation valable à prendre racine, ait échappé à cette sorte de fièvre de nature à sublimer les difficultés, les misères, l'horreur de la guerre indienne, voire le gâchis d'une existence mal orientée? Car du strict point de vue d'une colonisation qui a des chances de réussir, c'est bien, ici, de mauvaise orientation qu'il faut parler.

Quoi qu'il en soit, les institutions religieuses et sociales, elles, furent autant d'instruments chargés de maintenir cette vision d'un monde apostolique servant de pôle d'attraction au monde des infidèles. Il en fut ainsi des hôpitaux de Québec et de Montréal et du couvent des Ursulines. Sans doute devaient-ils aussi veiller aux besoins des colons français, mais ce n'était pas là leur tâche primordiale. Il suffit de lire les diverses annales de ces institutions pour s'en convaincre.

Dans cette conjoncture, l'Église comme telle, en 1632, ne pouvait être que résolument missionnaire. Elle l'était par sa juridiction qu'elle détenait de Rome. Elle l'était par ses préoccupations d'ouvrir des postes de mission de Tadoussac jusqu'en Huronie. Elle l'était par ses structures et son organisation qui plaçaient les colons français sous l'autorité spirituelle du supérieur des Jésuites dont le rôle essentiel consistait à répandre la doctrine catholique chez les infidèles.

Cependant, la colonie devait connaître des transformations qui allaient modifier l'orientation de ses institutions, y compris l’Église.

Comme terre de mission, la Nouvelle-France n'était pas viable. Elle avait pu susciter une immigration restreinte chez les humbles et, chez les grands, des générosités passagères. En France, le zèle se refroidissait rapidement ou se tournait vers d'autres œuvres. Témoin: la Société de Notre-Dame de Montréal. Au Canada, la destruction de la Huronie ruinait le plus clair des succès jésuites.

Le plus grave, c'est que l'idéal missionnaire, qui avait pu donner corps à une frêle colonie de peuplement, n'était jamais parvenu à assumer la vieille colonie d'exploitation des pelleteries 
qui continuait de tisser vers l'Ouest son réseau de trafic. Au cours de la décennie 1640-1650, le clivage et le manque d'unité sont évidents entre les deux entreprises de colonisation. Parce que la colonie de traite a déjà derrière elle une longue tradition, parce qu'elle s'adapte parfaitement à la réalité géo-économique, parce qu'elle est d'une organisation simple et peu coûteuse, parce qu'elle est rentable, enfin parce que le jour est encore loin où, pour elle, se poseront les problèmes difficiles de l'approvisionnement et des marchés rétrécis, c'est elle qui continue à monopoliser les aspects sains et prometteurs de la colonisation. C'est elle qui est responsable de la mise en place des premières structures socio-économiques et administratives qui dépassent tant soit peu le stade élémentaire des pouvoirs d'un gouverneur et d'un supérieur de jésuites sur un conglomérat de colons agricoles fraîchement débarqués sous l'empire d'une motivation éphémère. Par la création de la Communauté des Habitants en 1645 et par celle du Conseil de Traite en 1647, la NouvelleFrance donne l'image d'une société qui commence à s'organiser en tenant compte des modèles existants et des liens de subordination coloniale, mais surtout des lignes de force naturelles du milieu nord-américain où il lui fut donné de s'établir.

C'est cette Nouvelle-France, celle qu'anime l'empire de traite, qui réussira, par la Communauté des Habitants, à intégrer, provisoirement du moins, le petit nombre de colons que des idéaux élevés mais peu féconds continuaient d'attirer sur les bords du Saint-Laurent et à leur donner une nouvelle signification - pour ne pas dire une nouvelle mission - à partir du moment où ils perdront la leur.

La Communauté des Habitants devenait ainsi la Communauté des Français établis au Canada qui se préoccupaient avant tout de leur bien-être, de leur avenir, et pour qui l'Indien prenait un sens plus commercial qu'évangélique.

Cette réorientation de la colonie de peuplement devait amener une réévaluation des institutions déjà en place. Les structures de l'Église missionnaire notamment vont susciter des 
points d'interrogation. C'est que, dans la pensée gallicane, elles s'adaptent fort mal à une population française appelée à se développer en nombre et à former une société permanente qui vraisemblablement prendrait modèle sur la société mère.

Il nous apparaît assez révélateur qu'on ait soulevé la question d'un évêque pour le Canada au moment où se constituait la Communauté des Habitants. Deux ans plus tard, en 1647, les Jésuites de Québec cherchent à obtenir pour leur supérieur une juridiction gallicane dans le temps même où celui-ci s'apprête à siéger au Conseil de Traite.

Le mandat rouennais donné officiellement en 1648 établit les structures de base d'une Église gallicane spécialement chargée des besoins de la population française catholique (légitimité des professions religieuses, des mariages) et qui s'édifie parallèlement à l'Église missionnaire rattachée à Rome par les pouvoirs que détient le général des Jésuites.

Cette compartimentation subsiste jusqu'en 1659, par-delà les querelles qui opposent les Jésuites à Monsieur de Queylus et malgré les prétentions et de Rouen et de Rome d'occuper chacun la totalité du champ de juridiction. Elle témoigne d'une absence de droit reconnu et de jurisprudence en ce qui touche l'établissement des pouvoirs religieux dans une colonie à population française. Mais elle rend compte surtout, grâce justement à l'empirisme dans lequel elle est née, du double caractère qui marque la réalité coloniale, encore que l'aspect terre de mission soit déjà résolument à la baisse et en passe de devenir ce qu'il restera jusqu'à la fin du régime français, l'exercice d'une activité coloniale tout à fait secondaire.

En 1659, la colonie réclamait un évêque. Seules les structures d'un évêché régulier pouvaient, aux yeux de la métropole, régler la question de la double juridiction et encadrer d'une façon satisfaisante la population catholique et française de la vallée du Saint-Laurent.

Mais c'est d'un vicaire apostolique et non d'un évêque titulaire que la colonie hérita, signe évident de la faiblesse adminis- 
trative et diplomatique d'une France qui avait épuisé Mazarin et qui n'avait pas fini d'éduquer Louis XIV.

$\mathrm{Au}$ Canada, le vicariat apostolique perpétuait en principe la double juridiction, celle de Rome et celle de Rouen, de même que les querelles inévitables qu'elle continuait d'engendrer et qui occupaient pour une large part le temps et l'énergie des hommes d'Église et des chefs civils.

Mais ce qui importe davantage, c'est que le vicariat apostolique, par sa connotation missionnaire, mettait un accent indu sur ce qui n'était plus l'essentiel de l'Église coloniale et permettait par là à son titulaire - qui par sa formation n'en avait que trop tendance - de penser et d'orienter son institution dans un sens assez éloigné de celui du modèle gallican.

Nous sommes alors en 1663. Le problème de la double juridiction vient tout juste d'être tranché par un acte du pouvoir souverain. Le vicaire apostolique aura une autorité exclusive sur la colonie parce que, aux yeux du roi, il n'est plus vicaire apostolique mais déjà évêque titulaire d'un diocèse gallican qu'il appartient au pape de créer. Mais la décision royale ne porte pas immédiatement tous ses fruits. Notamment, elle ne peut faire que le vicariat apostolique, qui reste malgré tout le cadre officiel, s'inscrive dans le complexe de l'Église de France et en soit profondément influencé. D'ailleurs, jusqu'en 1665, il n'existe personne au Canada capable d'en faire une application rigoureuse. Le véritable représentant du roi dans la colonie, c'est le vicaire apostolique lui-même chargé d'entreprendre la réforme de l'administration civile mais en même temps libre d'organiser l'Église coloniale selon ses vues. A ce moment, il jouit de l'appui total du roi, sans pour autant subir la contrainte et la limitation de structures ecclésiales dont le modèle préétabli existe en France. Ce que l'on peut affirmer, c'est que, compte tenu de la direction dans laquelle la colonie était engagée, il a œuvré à contre-courant. La philosophie qui a inspiré la création du Séminaire de Québec en est la preuve la plus évidente. Entendons-nous: certaines dominantes administratives, comme la centralisation des effectifs matériels et 
humains, pouvaient correspondre à la conjoncture coloniale, bien circonstanciée, des années 1663-1665, mais l'ensemble de la politique de l'évêque s'insérait mal dans le courant évolutif rapide que connaissait alors la Nouvelle-France.

Cette évolution, qui s'accélère à l'époque de Talon, replaçait la colonie au niveau de l'idéal et de l'artificiel. Il ne s'agissait plus, comme en 1632, d'appliquer un programme arbitraire de missions, mais une politique de colonisation intégrale et intensive liée bien plus étroitement à un vaste plan mercantiliste qu'à la connaissance concrète de l'accessibilité et de la facilité d'exploitation des ressources de la colonie.

Un des préalables à cette politique, c'était de parfaire les rouages administratifs de la Nouvelle-France à partir du décalque de l'administration des provinces françaises sans trop se demander si la situation particulière du monde colonial ne nécessitait pas certaines adaptations ${ }^{1}$.

Cette optique allait amener la Cour et ses représentants officiels dans la colonie à pousser rapidement à ses limites la décision antérieure du roi de considérer le vicariat apostolique comme un évêché régulier. Il fallait modeler l'Église de la Nouvelle-France sur le prototype métropolitain ${ }^{2}$.

Pendant qu'on pressait Rome d'agir, dans la colonie on entreprenait la réorientation des structures de l'Église et d'abord de l'esprit qui les soustendait. En somme, la tâche consistait à décentraliser les foyers de décision en introduisant pour de bon l'État dans l'Église et en suscitant dans le monde religieux, selon un plan préétabli, des divergences d'opinions et de mentalités qui avaient pour effet d'affaiblir la pensée unanime qui dominait jusque-là.

1 Il y a une différence notable entre la genèse du Conseil de Traite de 1647 né de l'activité économique de la colonie et celle des institutions administratives créées à partir de 1663 sur des modèles métropolitains. Jusqu'à un certain point, cette différence caractérise les deux époques.

2 C'est encore la différence entre les besoins nés du milieu et l'application d'une politique a priori qui séparent les premières structures gallicanes de 1648 de celles qui s'élaborent sous Talon, visant à reproduire l'unité administrative du diocèse gallican. 
On avait à peine ébauché la réforme des structures, dont le règlement de la dîme en 1667 constitue la première démarche encore timide, quand l'évêché de Québec fut officiellement érigé. Le plus gros de la tâche de conversion structurale restait à faire. Mais déjà on pouvait affirmer que les cadres de l'Église étaient en voie de correspondre étroitement, sinon à la réalité coloniale qu'ils avaient peut-être dépassée en s'en inspirant trop peu, du moins, certes, à l'image curieusement déformée et résolument optimiste que, des bureaux de Colbert, on se faisait de la Nouvelle-France.

Université de Montréal

JEAN BLATN 